\title{
Bitcoin and Blockchain: Characteristics, Links and Implicit Potential for People and Markets
}

\section{Silvia Fernandes}

Faculty of Economics, University of Algarve, Campus de Gambelas, 8005-139 Faro, Portugal

\begin{abstract}
Named as the currency that would lead the monetary revolution, Bitcoin allows financial transactions without intermediation of banks that often charge high rates and are conducive to corruption threats. In Bitcoin, the intermediary is eliminated and with it its weaknesses. This perspective responds to the influence that corruption and mismanagement of banks and governments have on the economy (e.g. inflation).
\end{abstract}

Bitcoin is still more effective if integrated into a Blockchain network that allows transactions to be verified by all network users (nodes). When committed, transactions are recorded in an accounting 'diary' that is synchronized between all users [1]. From there comes another feature that makes this digital currency more interesting, which is the transparency of information made available to all in a transversal way. The information flows in a decentralized manner, making it unfeasible for any financial or governmental authority to manipulate its emission or its value, causing inflation or financial collapse (similarly to cases such as the September of 2008).

Blockchain may help a country's financial future by increasing process transparency and the confidence of millions of users who daily move money [2]. It functions as an accounting diary, the content of which is public and freely accessible to all network users. All transactions occurring in the network, once verified, are recorded in this structure [3]. The 'guarantee' of transparency in transactions

\section{Publication History:}

Received: June 20, 2019

Accepted: July 25, 2019

Published: July 27, 2019

\section{Keywords:}

IoT, Security, Security framework, Review, Mobile edge computing

first to validate the transfers and consequently to receive the reward. Once a user validates this operation, all others synchronize the diary's entry and start searching for the key of the next transaction. These individuals protect the transactions in the structure [1].

The mining is done by means of great computational force that works in the sense of quickly authenticate the transactions. Thus, there are users who are profitable because they have access to low-cost hardware and electricity, and can thus capitalize on this opportunity. The miners are mostly decentralized, as they look for areas where the price of electricity is lower, around several countries. This makes it even more costly to defraud the system, not worth influencing such a computational force [4]. Blockchain technology gives to bitcoin credibility and, in a framework without intermediaries, ensures that transactions are carried out by reducing the need for trust among the agents involved, a considerable pressure in today's relation between clients and banks.

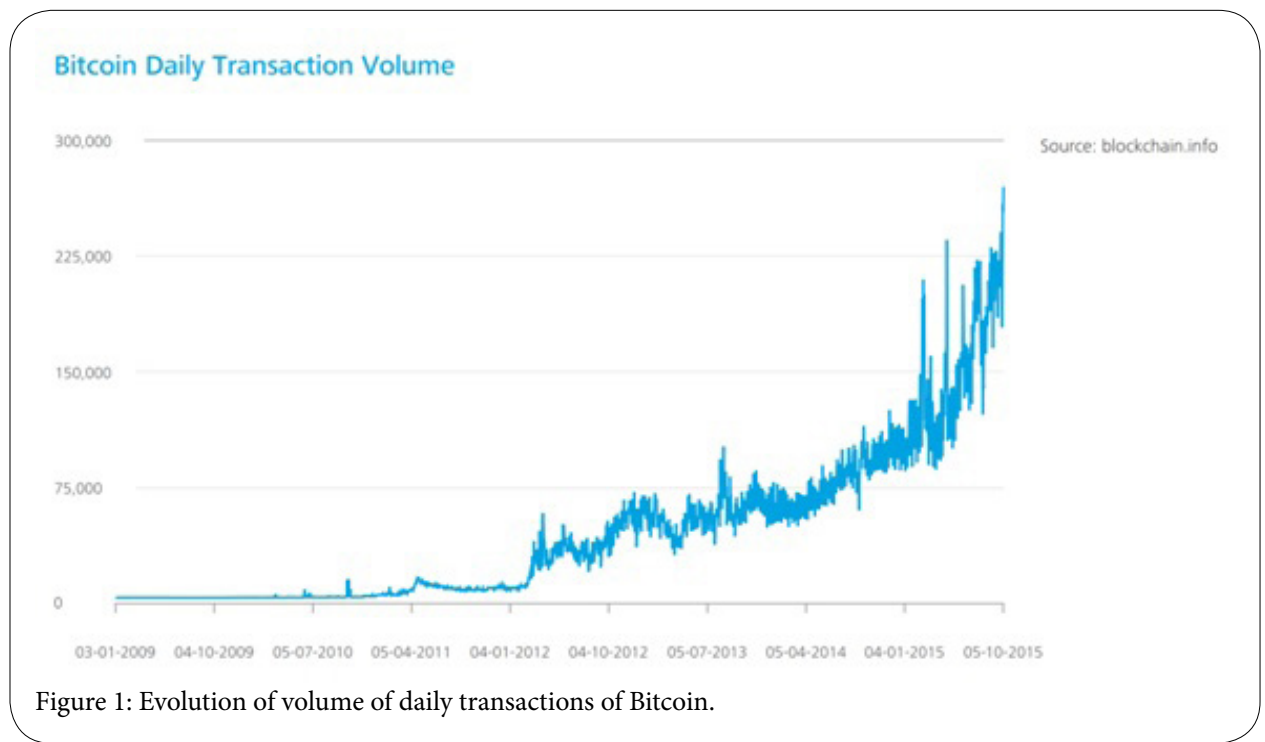

is possible because this diary is written together, synchronized between all parties, so that all participants can constantly confirm the information and monitor who owns a certain amount.

Confirming the reliability of the transfers depends on the speed of who actively validates the transactions. The more users try to validate transactions, the faster the currency mobility will be. To encourage users to do so, the system rewards those who can be the first to validate the transfer and put it as an entry in the accounting diary. These users are called 'miners' because they compete with each other to be the

\begin{abstract}
"Corresponding Author: Dr. Silvia Fernande, Faculty of Economics, University of Algarve, Campus de Gambelas, 8005-139 Faro, Portugal; E-mail: faivlis.fernandes@gmail.com
\end{abstract}

Citation: Fernandes S (2019) Bitcoin and Blockchain: Characteristics, Links and Implicit Potential for People and Markets. Int J Comput Softw Eng 4: 149. doi: https://doi.org/10.15344/2456-4451/2019/149

Copyright: (c) 2019 Fernande. This is an open-access article distributed under the terms of the Creative Commons Attribution License, which permits unrestricted use, distribution, and reproduction in any medium, provided the original author and source are credited. 
Citation: Fernandes S (2019) Bitcoin and Blockchain: Characteristics, Links and Implicit Potential for People and Markets. Int J Comput Softw Eng 4: 149. doi: https://doi.org/10.15344/2456-4451/2019/149

Page of

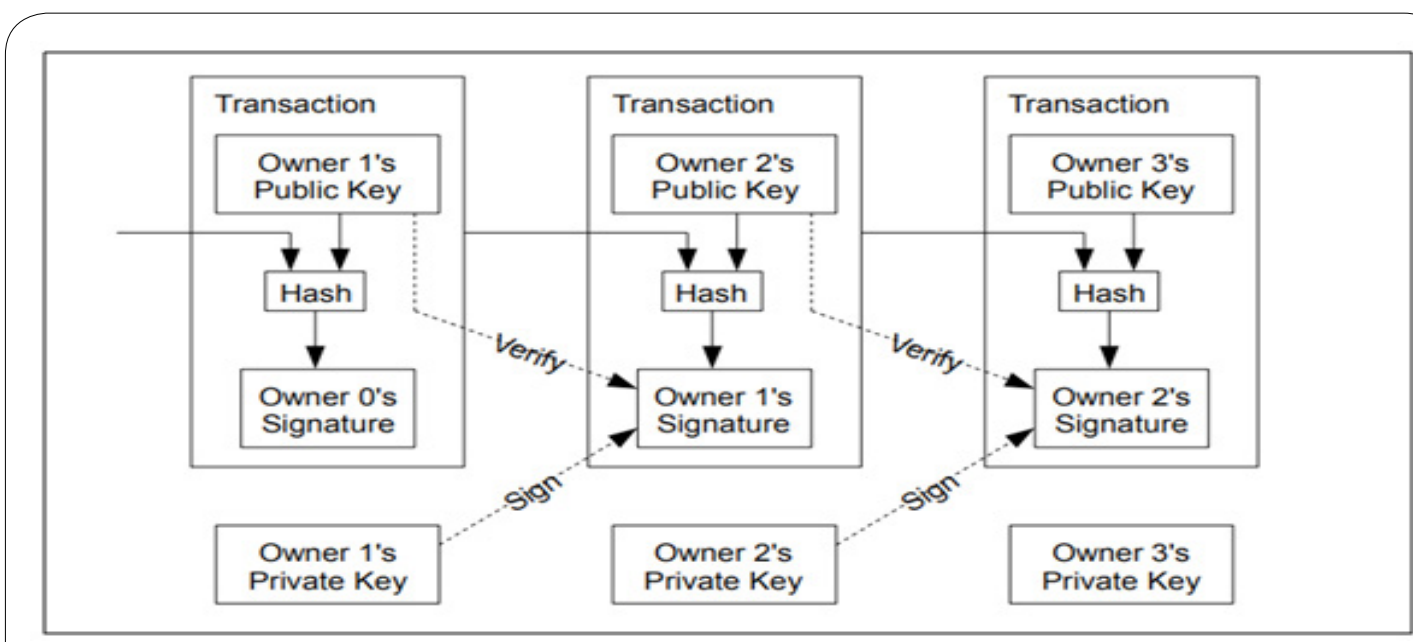

Figure 2: Blockchain transaction network.

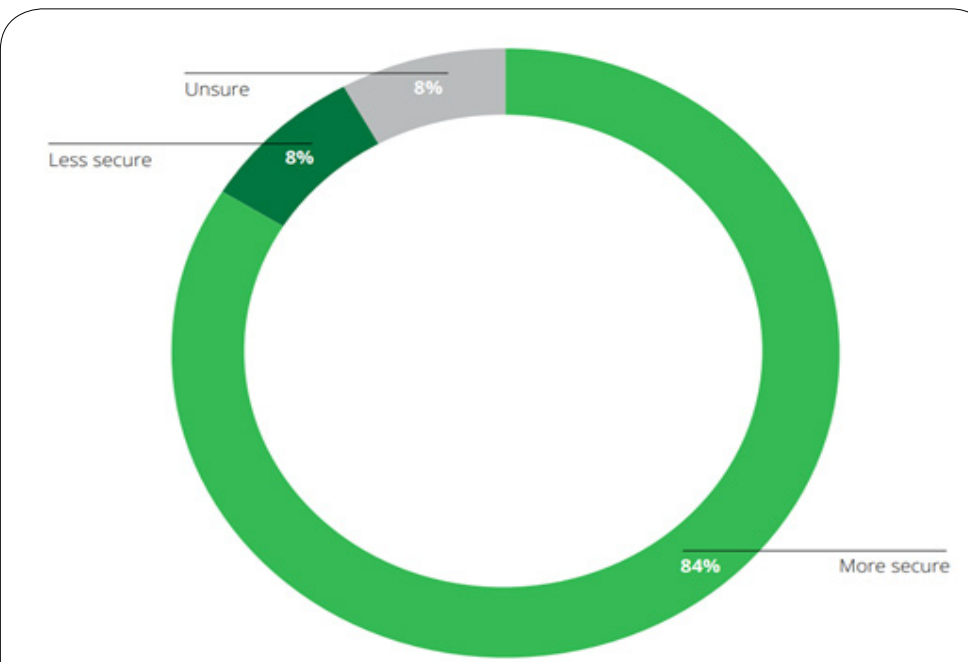

Figure 3: Answers to the question: "Is Blockchain a safer system when compared to conventional technologies?"

One study shows that $84 \%$ of people believe that Blockchain is safer than the traditional methods applied by banks in their closed-loop transactions [5].

Bitcoin has had an undeniable impact on current society and current managers. It was the first crypto-currency that really got in the way of how we currently trade currency. Blockchain technology can remove much inefficiency from the current banking system, reducing the associated costs. Banks are thinking of a way to bring Wall Street to the Bitcoin. The idea is to make it possible to use this technology and create its own Blockchain network, not with anonymous miners, but with computers controlled by them. They are thus experimenting with ways of including this system in their institutions, not only in currency transactions, but in stock trading, patents, etc. (Banking on Bitcoin - Netflix).

Some businesses and companies already offer this new currency as a mean of payment. In Portugal there are already 24 million accounts that trade it [6]. Today we know that there is the possibility of an economy without intermediaries, independent from banks and government, where trust is no longer a critical element. Therefore, Blockchain provided considerable changes in the way the currency transactions take place: 1) giving total anonymity to users; 2) controlling data and information through them; and 3) ceasing to be processed by large centralizers.

Competing Interests

The author declare that there is no competing interests regarding the publication of this article.

\section{References}

1. Nakamoto S (2008) Bitcoin: A peer-to-peer electronic cash system.

2. Amaro MS (2018) Bitcoin, criptomoedas e blockchain: Oportunidade ou bolha?.

3. Pacheco L, Tavares F, Araújo B (2018) A Bitcoin e o seu desenvolvimento: Estudo aplicado a uma amostra representativa.

4. TechCrunch (2016) Mines and miners: Trust disrupted bitcoin and the Blockchain. https://youtu.be/FtR06bIDxkE

5. Deloitte (2018) Global blockchain survey.

6. Observador (2019) Jovens adultos do sexo masculino são quem mais investe em 'bitcoin'. 